\title{
Broadening and shift of Rydberg levels by elastic collisions with rare-gas atoms
}

\author{
B Kaulakys \\ Institute of Physics of the Academy of Sciences of the Lithuanian SSR, 232600 Vilnius, \\ USSR
}

Received 15 February 1984, in final form 21 June 1984

\begin{abstract}
We obtain expressions for Rydberg level broadening and shift cross sections and rates suitable for elastic collisions of ground-state rare-gas atoms with Rydberg atoms. The semiclassical impact broadening theory is used. The interaction of a Rydberg atom with a rare-gas atom is approximated by the superposition of polarisation potentials and the Fermi pseudopotential. It is shown that similar expressions for the broadening and shift cross sections due to the perturber-Rydberg-electron interaction can be obtained in the JWKB approximation for the Rydberg-electron wavefunctions and for the square-well model potential. Consequently the shift and width of the Rydberg level are not very sensitive to the shape of the potential but only to its normalisation. Expressions for the shift and width in the square-well potential approximation suitable in the high-density limit of the perturbing atoms are obtained. Limits of the applicability of the expressions for the broadening and shift cross sections are discussed. Calculated broadening and shift rates agree well with experimental results for the broadening and shift of Rydberg levels with small, medium and large principal quantum numbers by all rare gases excepting neon.
\end{abstract}

\section{Introduction}

Measurements of the shift and broadening of spectral lines associated with transitions between the ground state and highly excited states have been performed continually during the last fifty years and are being carried out at present. A review of early work in this field can be found in the paper by Ch'en and Takeo (1957). An analysis of later experimental and theoretical investigations of the broadening and shift of atomic Rydberg levels in gases is given in the review by Kaulakys and Serapinas (1984). The theory of the interaction and collisions of Rydberg atoms with neutral perturbers, first formulated by Fermi (1934) and improved by Reinsberg (1937), Firsov (1951), Alekseev and Sobel'man (1965), has been recently reconsidered by Omont (1977).

On the basis of the paper by Alekseev and Sobel'man (1965) the broadening and shift of a high-Rydberg series perturbed by neutral atomic particles were related to the resonances in the elastic scattering of an extremely slow electron by the perturber (Matsuzawa 1975, 1977, Kaulakys et al 1979, Kaulakys 1980, 1982). This allowed us to explain the oscillations in the dependences of the widths and shifts on the principal quantum number of the Rydberg levels of alkali atoms in the self-broadening. From a comparison of theory with experimental data (Mazing and Serapinas 1971, Stoicheff and Weinberger 1980, Stoicheff et al 1981) the energy and width of the ${ }^{3} \mathrm{P}$ resonance 
in the scattering of an electron by alkali ground-state atoms was obtained (Kaulakys 1982). The existence of this resonance explains the considerably stronger broadening of the alkali atoms' Rydberg levels in self-broadening than in the broadening by noble gas (Kaulakys 1980).

However the theory of Alekseev and Sobel'man (1965), as was shown by Omont (1977), is valid only under the conditions, where the Rydberg atom undergoes essentially inelastic collisions. For collisions with noble-gas atoms this means $n^{2} \gg v^{-1}$, where $n$ is the principal quantum number of the Rydberg level and $v$ is the collision velocity. For collisions with other atoms, e.g. alkalis, the validity condition of the theory of Alekseev and Sobel'man cannot be so strong. In the case when the theory of Alekseev and Sobel'man (1965) fails the classical-path method for the description of the groundstate atom collisions with Rydberg atoms can be used. For elastic collisions the broadening and shift cross sections can be expressed in terms of the semiclassical phaseshift. However, in the case of small and medium $n$ the contributions to the broadening and shift cross sections due to the Rydberg-electron-perturber and Rydberg-atom-core-perturber interactions are not additive. These cross sections must be calculated using the phaseshift due to both interactions (Omont 1977). Broadening cross sections were calculated numerically in such a way by Ueda et al (1982a, b) for rare-gas-induced broadening of calcium principal series lines.

The purpose of the present work is to obtain expressions for the Rydberg level broadening and shift cross sections due to the elastic collisions of Rydberg atoms with ground-state noble-gas atoms of low and high densities. In addition, the comparison between theory and experimental data will be presented and validity criterion of the theory will be discussed.

We make the following assumptions: (i) the impact approximation of the spectral line-broadening theory is suitable for low densities of the perturbing atoms, (ii) the perturbation of the upper (Rydberg) state induces the line broadening and shift, (iii) the semiclassical theory and straight-line trajectory approximation are appropriate, (iv) the interaction of a Rydberg atom with a rare-gas atom is approximated by a superposition of polarisation potentials and the Fermi pseudopotential, $(v)$ the line broadening is induced by the isotropic part of these potentials, (vi) the JWKB approximation for the Rydberg-electron wavefunctions is appropriate, and (vii) contribution from inelastic collisions to the broadening is negligible.

\section{Impact approximation for broadening and shift of Rydberg levels}

According to the impact theory, the intensity distribution of the line corresponding to a transition between two levels is given by the Lorentzian profile. The width $\gamma$ and the shift $\Delta$ of the line can be written as (see, e.g. Sobel'man et al 1981)

$$
\gamma=2 N\left\langle\sigma^{\prime} v\right\rangle \quad \Delta=N\left\langle\sigma^{\prime \prime} v\right\rangle
$$

where $N$ is the perturber density, $\sigma^{\prime}$ and $\sigma^{\prime \prime}$ are the effective cross sections for the impact broadening and shift of the line, respectively. The average is taken over the distribution of relative velocity of the interacting atoms. It should be emphasised that the broadening and shift of an optical line corresponding to the transition between the Rydberg state and the low-lying state (e.g. ground state) is entirely determined by the perturbation of the Rydberg state. 
For elastic Rydberg-atom-perturber collisions the broadening and shift cross sections can be written as

$$
\begin{aligned}
& \sigma^{\prime}=2 \pi \int_{0}^{\infty}(1-\cos \eta(\rho)) \rho \mathrm{d} \rho \\
& \sigma^{\prime \prime}=-2 \pi \int_{0}^{\infty} \sin \eta(\rho) \rho \mathrm{d} \rho
\end{aligned}
$$

where $\rho$ is the impact parameter and $\eta$ is the semiclassical phaseshift due to the total interaction $V$ between the Rydberg atom and perturber. Here $\eta$ is given by the formula

$$
\eta(\rho)=-\frac{2}{v} \int_{\rho}^{\infty} \frac{V(\boldsymbol{R})}{\left(1-\rho^{2} / R^{2}\right)^{1 / 2}} \mathrm{~d} R
$$

where $R$ is the internuclear distance. A straight-line trajectory in equation (4) is assumed.

\section{Interaction of a Rydberg atom with a neutral atomic particle}

The potential of the interaction between the Rydberg atom and the neutral atomic particle consists of the polarisation attractions and the short-range interaction between the Rydberg electron and perturber. The polarisation interaction $V_{\mathrm{pol}}=-\frac{1}{2} \alpha E^{2}$, where $\alpha$ is the polarisability of the perturber and

$$
\boldsymbol{E}=\frac{\boldsymbol{R}}{\boldsymbol{R}^{3}}-\frac{\boldsymbol{R}-\boldsymbol{r}}{|\boldsymbol{R}-\boldsymbol{r}|^{3}}, \quad|\boldsymbol{R}-\boldsymbol{r}|>r_{0}
$$

is the electric field originated by the core of the Rydberg atom and the Rydberg electron. Here $\boldsymbol{R}$ and $\boldsymbol{r}$ are the location of the perturber and the coordinate of the electron, respectively, $r_{0}$ is the distance of the short-range interaction between the electron and perturber. Thus

$$
V_{\mathrm{pol}}(\boldsymbol{R}, \boldsymbol{r})=-\frac{\alpha}{2 R^{4}}+\alpha \frac{\boldsymbol{R}^{2}-(\boldsymbol{R} \cdot \boldsymbol{r})}{R^{3}|\boldsymbol{R}-\boldsymbol{r}|^{3}}-\frac{\alpha}{2|\boldsymbol{R}-\boldsymbol{r}|^{4}} \quad|\boldsymbol{R}-\boldsymbol{r}|>r_{0} .
$$

$V_{\mathrm{c}}(R)=-\alpha / 2 R^{4}$ is the polarisation attraction between the perturber and the core of the Rydberg atom,

$$
V_{\mathrm{ce}}=\alpha \frac{R^{2}-(\boldsymbol{R} \cdot \boldsymbol{r})}{R^{3}|\boldsymbol{R}-\boldsymbol{r}|^{3}}
$$

is the interaction between the Rydberg electron and dipole momentum of the perturber induced by the core of the Rydberg atom. The calculation of the matrix element of the operator $V_{\mathrm{ce}}$ becomes simpler if the expansion of this operator in terms of the Legendre functions $P_{\kappa}$ is used. Then equation (6) is simply

$$
V_{\mathrm{ce}}(\boldsymbol{R}, \boldsymbol{r})=\sum_{\kappa} V_{\mathrm{ce}}^{(\kappa)}(R, r) P_{\kappa}\left(\frac{\boldsymbol{R} \cdot \boldsymbol{r}}{R r}\right)
$$


where the expansion coefficient in (7) is (see Baylis 1969)

$$
V_{\mathrm{ce}}^{(\kappa)}(R, r)= \begin{cases}\alpha \frac{\kappa+1}{R^{4}}\left(\frac{r}{R}\right)^{\kappa} & r<R \\ -\alpha \frac{\kappa}{R^{3} r}\left(\frac{R}{r}\right)^{\kappa} & r>R .\end{cases}
$$

The potential of the electron-perturber interaction $V_{\mathrm{e}}(\boldsymbol{r}-\boldsymbol{R})$ consists of the polarisation attraction $-\alpha / 2|\boldsymbol{r}-\boldsymbol{R}|^{4}$. and short-range interaction. This potential causes the electron-atom scattering cross section and, for an atom with small polarisability, may be approximated by a Fermi pseudopotential

$$
V_{\mathrm{e}}(\boldsymbol{r}-\boldsymbol{R})=2 \pi L \delta(\boldsymbol{r}-\boldsymbol{R})
$$

where $L$ is the scattering length.

Using hydrogen-atom wavefunctions

$$
\psi_{n l m}(\boldsymbol{r})=R_{n l}(r) Y_{l m}(\boldsymbol{r} / \boldsymbol{r})
$$

we may evaluate the matrix elements of the operator $V$

$$
\begin{aligned}
\left\langle n_{1} l_{1} m_{1}|V| n_{2} l_{2} m_{2}\right\rangle & \\
= & -\frac{\alpha}{2 R^{4}} \delta_{n_{1} n_{2}} \delta_{l_{1} l_{2}} \delta_{m_{1} m_{2}}+\sum_{\kappa}\left\langle l_{1} m_{1}\left|P_{\kappa}\right| l_{2} m_{1}\right\rangle \delta_{m_{1} m_{2}} \\
& \times \int_{0}^{\infty} V_{\mathrm{ce}}^{(\kappa)}(R, r) R_{n_{1} l_{1}}(r) R_{n_{2} l_{2}}(r) r^{2} \mathrm{~d} r+2 \pi L \psi_{n_{1} l_{1} m_{1}}^{*}(\boldsymbol{R}) \psi_{n_{2} l_{2} m_{2}}(\boldsymbol{R}) .
\end{aligned}
$$

The matrix element $\left\langle l_{1} m\left|P_{\kappa}\right| l_{2} m\right\rangle$ can be expressed in terms of $3-j$ symbols and evaluated, e.g.

$$
\begin{aligned}
& \left\langle l m\left|P_{0}\right| l m\right\rangle=1 \\
& \left\langle\operatorname{lm}\left|P_{1}\right| l+1 m\right\rangle=\left(\frac{(l+1)^{2}-m^{2}}{(2 l+1)(2 l+3)}\right)^{1 / 2} \\
& \left\langle\operatorname{lm}\left|P_{2}\right| l m\right\rangle=\frac{l(l+1)-3 m^{2}}{(2 l-1)(2 l+3)} \quad l \geqslant 1
\end{aligned}
$$

and so on.

When only elastic collisions are important one needs only the diagonal matrix elements of the operator $V$

$$
\begin{aligned}
V_{n l m}(\boldsymbol{R}) \equiv & \langle n l m|V| n l m\rangle \\
& =-\frac{\alpha}{2 R^{4}}+\sum_{\kappa}\left\langle\operatorname{lm}\left|P_{\kappa}\right| l m\right\rangle \int_{0}^{\infty} V_{\mathrm{ce}}^{(\kappa)}(R, r) R_{n l}^{2}(r) r^{2} \mathrm{~d} r+2 \pi L R_{n l}^{2}(R)\left|Y_{l m}(\boldsymbol{R} / R)\right|^{2} .
\end{aligned}
$$

The isotropic part of this matrix element, or matrix element averaged over magnetic quantum number, is

$$
V_{n l}(R) \equiv \frac{1}{2 l+1} \sum_{m} V_{n l m}(\boldsymbol{R})=-\frac{\alpha}{2 R^{4}}+\frac{\alpha}{R^{4}} \int_{0}^{R} R_{n l}^{2}(r) r^{2} \mathrm{~d} r+\frac{1}{2} L R_{n l}^{2}(R) .
$$


This averaging in the calculation of the phaseshift according to equation (4) means that the quantisation axis of the Rydberg states is not rotated in the perturber-Rydbergatom collisions.

For evaluation of the integral in equations (12)-(13) the JWKB approximation for the radial parts of the wavefunctions is convenient to use. We can write, according to Omont (1977),

$$
R_{n i}(r)= \begin{cases}\left(\frac{2}{\pi n^{* 3}}\right)^{1 / 2} \frac{1}{r \sqrt{p_{r}}} \sin \left(\int_{r_{1}}^{r} p_{r} \mathrm{~d} r+\frac{1}{4} \pi\right) & r_{1}<r<r_{2} \\ 0 & r \leqslant r_{1}, r \geqslant r_{2}\end{cases}
$$

where $p_{r}$ is the radial impulse,

$$
p_{r}=\left[2 / r-1 / n^{* 2}-\left(l+\frac{1}{2}\right)^{2} / r^{2}\right]^{1 / 2}
$$

$r_{1}$ and $r_{2}$ are the roots of $p_{r}$ which delimit the region of classical motion,

$$
r_{1,2}=n^{* 2} \neq n^{*}\left[n^{* 2}-\left(l+\frac{1}{2}\right)^{2}\right]^{1 / 2}
$$

and $n^{*}$ is the effective principal quantum number.

\section{Broadening and shift cross sections}

According to equations (4) and (5) the phaseshift $\eta$ may be written as

$$
\eta(\rho)=\eta_{\mathrm{c}}(\rho)+\eta_{\mathrm{ce}}(\rho)+\eta_{\mathrm{e}}(\rho)
$$

where

$$
\eta_{\mathrm{c}}(\rho)=\frac{\pi \alpha}{4 v \rho^{3}}
$$

is the phaseshift due to the polarisation attraction between the perturber and core of the Rydberg atom, $\eta_{\mathrm{e}}$ and $\eta_{\mathrm{ce}}$ are the phaseshifts caused by Rydberg-electron-perturber interaction and by the potential $V_{\mathrm{ce}}(R)$ (see equation (6)), respectively. In the case of the Fermi pseudopotential $\eta_{\mathrm{e}}$ can be evaluated according equations (4), (13) and (14)

$$
\begin{aligned}
\eta_{\mathrm{e}}(\rho) & =-\frac{L}{\pi v n^{* 2}} \int_{\rho}^{r_{2}} \frac{\mathrm{d} r}{\left[\left(r_{2}-r\right)\left(r-r_{1}\right)\left(r^{2}-\rho^{2}\right)\right]^{1 / 2}} \\
& =-\frac{2 L K(r)}{\pi v n^{* 2}\left[2\left(r_{2}-r_{1}\right) \rho\right]^{1 / 2}} \quad r_{1}<\rho<r_{2} \\
r & =\left(\frac{\left(r_{2}-\rho\right)\left(r_{1}+\rho\right)}{2\left(r_{2}-r_{1}\right) \rho}\right)^{1 / 2}
\end{aligned}
$$

where $K$ is the complete elliptic integral of the first kind. In equation (18) $\sin ^{2}(\ldots)$ have been replaced by $\frac{1}{2}$. It can be seen that $K$ approximately equals $\pi / 2$ if $1 \ll n$. Accordingly

$$
\eta_{\mathrm{e}}(\rho)=-\frac{L}{2 v n^{* 3} \sqrt{\rho}} \quad r_{1}<\rho<r_{2} .
$$


$\eta_{\mathrm{ce}}$ may be evaluated in the same way. We have

$$
\eta_{\mathrm{ce}}(\rho) \simeq \begin{cases}-\frac{\alpha}{v \rho^{3}}\left(\frac{\pi}{4}-\frac{1}{2} \sin ^{-1} \frac{r_{2}-2 \rho}{r_{2}}-\frac{\left[\rho\left(r_{2}-\rho\right)\right]^{1 / 2}}{r_{2}}\right) & \rho \leqslant \frac{1}{2} r_{2} \\ -\frac{\pi \alpha}{2 v \rho^{3}}+\frac{\alpha\left(r_{2}^{2}+\rho^{2}\right)\left(r_{2}-\rho\right)}{v r_{2}^{2} \rho^{4}}\left(\frac{r_{2}+\rho}{r_{2}}\right)^{1 / 2} & \frac{1}{2} r_{2} \leqslant \rho \leqslant r_{2} \\ -\frac{\pi \alpha}{2 v \rho^{3}} & \rho \geqslant r_{2} .\end{cases}
$$

Thus $\left|\eta_{\mathrm{ce}}\right| \ll\left|\eta_{\mathrm{c}}\right|$ if $\rho \leqslant \frac{1}{2} r_{2}$. It should be noted that for $R>r_{2}$ the polarisation interaction between the perturber and Rydberg atom defined by equation (5) results the van der Waals potential

$$
V_{\mathrm{pol}}^{(0)}(\boldsymbol{R}, \boldsymbol{r})=-\frac{\alpha r^{2}}{R^{6}}\left[1+2\left(\frac{r}{R}\right)^{2}+\ldots\right] \quad r<R .
$$

Approximately the total phaseshift may be written as

$$
\eta(\rho) \simeq \begin{cases}\frac{\pi}{4} \frac{\alpha}{v \rho^{3}}-\frac{L}{2 n^{* 3} v \sqrt{\rho}} & \rho \leqslant n^{* 2} \\ -\frac{L}{2 n^{* 3} v \sqrt{\rho}} & n^{* 2} \leqslant \rho<2 n^{* 2} \\ \frac{15 \pi \alpha n^{* 4}}{16 v \rho^{5}} & \rho>2 n^{* 2} .\end{cases}
$$

The broadening and shift cross sections can be calculated substituting equation (22) into equations ( 2 ) and (3). It should be emphasised that Rydberg-atom-perturber collisions with $\rho>2 n^{* 2}$ cause small contributions to the broadening and shift cross sections. For the van der Waals potential this can be seen from equations (2), (3) and (22), while for the Fermi pseudopotential $V_{\mathrm{e}}(\boldsymbol{R})=2 \pi L\left|\psi_{n l m}(\boldsymbol{R})\right|^{2}$ (see equation (9)) this follows from the analysis of the asymptotic of the radial part of the wavefunction which can be written as

$$
R_{n l}^{2}(r)=\frac{2^{2 / 3} \Phi^{2}(\xi)}{\pi n^{* 3} r_{2}^{4 / 3}} \quad \xi=\left(\frac{2}{r_{2}^{2}}\right)^{1 / 3}\left(r_{2}-r\right) \quad r \sim r_{2}
$$

where $\Phi$ is the Airy function (see Landau and Lifshitz 1960).

For small $n^{*}$ the integrals in equations (2)-(3) are defined by $\eta_{\mathrm{e}}$. Accordingly we have

where

$$
\begin{aligned}
& \sigma_{\mathrm{e}}^{\prime}=4 \pi n^{* 4}+\frac{2}{3} \pi n^{* 4}\left[x^{4} \operatorname{Ci}(x)-\left(x^{3}-2 x\right) \sin x-\left(6-x^{2}\right) \cos x\right] \\
& n^{*}<2^{-4 / 9}(\pi / 5.7)^{1 / 12} n_{2}^{*} \simeq 0.70 n_{2}^{*} \\
& \sigma_{\mathrm{e}}^{\prime \prime}=\frac{2 \pi L}{v} \frac{1}{6 \sqrt{2}}\left[\left(2-x^{2}\right) \cos x+\left(\frac{6}{x}-x\right) \sin x-x^{3} \operatorname{Si}(x)+\frac{1}{2} \pi x^{3}\right] \\
& n^{*}<2^{1 / 8} n_{1}^{*} \simeq 1.09 n_{1}^{*}
\end{aligned}
$$

$$
x=2^{1 / 2}\left(\frac{n_{1}^{*}}{n^{*}}\right)^{4} \quad n_{1}^{*}=\left(\frac{|L|}{4 v}\right)^{1 / 4} \quad n_{2}^{*}=\left(\frac{|L|}{\alpha^{1 / 6} v^{5 / 6}}\right)^{1 / 3}
$$


$\mathrm{Si}(x)$ and $\mathrm{Ci}(x)$ are the sine and cosine integrals. From equations (23) and (24) it follows

$$
\begin{aligned}
\sigma_{\mathrm{e}}^{\prime} & \simeq \begin{cases}4 \pi n^{* 4} & n^{*}<n_{1}^{*} \\
\frac{8 \pi n_{1}^{* 8}}{n^{* 4}}\left(1-\frac{n_{1}^{* 8}}{2 n^{* 8}}\right) & n_{1}^{*}<n^{*}<0.70 n_{2}^{*}\end{cases} \\
\sigma_{\mathrm{e}}^{\prime \prime} & \simeq 0 \quad n^{*}<1.09 n_{1}^{*} .
\end{aligned}
$$

For larger $n^{*}$ from equations (2), (3) and (22) we have

$$
\begin{aligned}
& \sigma^{\prime}=\sigma_{\mathrm{c}}^{\prime}+\frac{8 \pi n_{1}^{* 8}}{n^{* 4}}\left[1-\left(\frac{5.7}{\pi}\right)^{1 / 2}\left(\frac{\alpha}{2 v}\right)^{1 / 3} \frac{1}{2 n^{* 2}}\right] n^{*}>0.70 n_{2}^{*} \\
& \sigma^{\prime \prime}= \begin{cases}\frac{2 \pi L}{v}\left(1-\frac{n_{1}^{* 8}}{n^{* 8}}\right)+\sigma_{\mathrm{c}}^{\prime \prime}\left(\frac{\alpha}{2 v}\right)^{1 / 3}\left(\frac{n^{* 6}}{2 n_{1}^{* 8}}-\frac{1}{n^{* 2}}\right) & 1.09 n_{1}^{*}<n^{*}<2^{-4 / 9} n_{2}^{*} \simeq 0.73 n_{2}^{*} \\
\frac{2 \pi L}{v}\left(1-\frac{n_{1}^{* 8}}{n^{* 8}}\right)+\sigma_{\mathrm{c}}^{\prime \prime}\left[1-\left(\frac{\alpha}{2 v}\right)^{1 / 3} \frac{1}{n^{* 2}}\right] & n^{*}>0.73 n_{2}^{*}\end{cases}
\end{aligned}
$$

where

$$
\sigma_{\mathrm{c}}^{\prime}=\left(\frac{\pi}{2}\right)^{5 / 3} \Gamma\left(\frac{1}{3}\right)\left(\frac{\alpha}{2 v}\right)^{2 / 3} \simeq 5.7\left(\frac{\alpha}{2 v}\right)^{2 / 3} \quad \sigma_{\mathrm{c}}^{\prime \prime}=-\sqrt{3} \sigma_{\mathrm{c}}^{\prime}
$$

are the broadening and shift cross sections due to the polarisation interaction between the perturber and core of the Rydberg atom. In equation (29) $\frac{2}{3} \sqrt{2}$ is replaced by 1 .

Thus we have that the broadening cross section as a function of $n^{*}$ for small $n^{*}$ increases as $n^{* 4}$, reaches its maximum value $\sigma_{\max }^{\prime}=\frac{16}{3} \sqrt{\frac{2}{3}} \pi n_{1}^{* 4}$ at $n^{*}=\left(\frac{3}{2}\right)^{1 / 8} n_{1}^{*}$ and then decreases asymptotically approaching the value $\sigma_{\mathrm{c}}^{\prime}$. The shift cross section increases very rapidly at $n^{*} \simeq n_{1}^{*}$, and reaches its asymptotic value $\sigma^{\prime \prime}=\sigma_{\mathrm{e}}^{\prime \prime}+\sigma_{\mathrm{c}}^{\prime \prime}$, where $\sigma_{\mathrm{e}}^{\prime \prime}=$ $2 \pi L / v$. Note that equation (28) is close to the expression given by Omont (1977).

It is important to note, that the broadening and shift cross sections due to the perturber-Rydberg-electron interaction are not very sensitive to the shape of the potential but only to its normalisation. To show this we consider scattering by the square-well potential

$$
V_{\text {sw }}(R)= \begin{cases}2 \pi L / \frac{4}{3} \pi r_{2}^{3}=3 L / 16 n^{* 6} & R<2 n^{* 2} \\ 0 & R>2 n^{* 2}\end{cases}
$$

(see Hickman 1979). The normalisation is chosen such that $4 \pi \int_{0}^{\infty} V_{\mathrm{sw}}(R) R^{2} \mathrm{~d} R=2 \pi L$. Potential (31) corresponds to the Fermi pseudopotential (9) when the electron charge density is uniformly spread out inside a sphere of radius $r_{2}$. Substituting equation (31) into equations (2)-(4) we obtain expressions for the broadening and shift cross sections which are very close to the expressions (22) and (24). Approximately

$$
\begin{gathered}
\sigma_{\mathrm{sw}}^{\prime}= \begin{cases}4 \pi n^{* 4} & n^{*}<n_{1}^{*} \\
\frac{9 \pi n_{1}^{* 8}}{n^{* 4}}\left(1-\frac{n_{1}^{* 8}}{2 n^{* 8}}\right) & n^{*}>n_{1}^{*}\end{cases} \\
\sigma_{\mathrm{sw}}^{\prime \prime}= \begin{cases}0 & n^{*} \leqslant n_{1}^{*} \\
\frac{2 \pi L}{v}\left(1-\frac{9}{10} \frac{n_{1}^{* 8}}{n^{* 8}}\right) & n^{*} \geqslant n_{1}^{*} .\end{cases}
\end{gathered}
$$

Compare equations (32)-(33) with equations (26)-(29). 
Broadening and shift rates may be expressed in terms of the averaged cross sections according to equation (1):

$$
\sigma_{v}^{\prime}=\left\langle\sigma^{\prime} v\right\rangle / \bar{v} \quad \sigma_{v}^{\prime \prime}=\left\langle\sigma^{\prime \prime} v\right\rangle / \bar{v} .
$$

For a Maxwellian distribution of relative velocity of the colliding atoms from equations (26), (28), (30) we have

$$
\sigma_{v}^{\prime}= \begin{cases}4 \pi n^{* 4} & n^{*}<n_{1}^{*} \\ \frac{2 \sqrt{\pi}|L|}{\bar{v}} f(y) & n^{*} \leqslant 0.70 n_{2}^{*} \\ 5.5\left(\frac{\alpha}{2 \bar{v}}\right)^{2 / 3}+\frac{2 L^{2}}{\bar{v}^{2} n^{* 4}}-\frac{1.7 L^{2}}{\bar{v}^{2} n^{* 6}}\left(\frac{\alpha}{2 \bar{v}}\right)^{1 / 3} & n^{*} \geqslant n_{2}^{*}\end{cases}
$$

where

$$
\begin{aligned}
& \bar{v}=\left(\frac{8 k T}{\pi \mu}\right)^{1 / 2} \quad y=\frac{L^{2}}{4 \pi \bar{v}^{2} n^{* 8}} \\
& f(y)=y^{-1 / 2}\left[1-(1-y) \mathrm{e}^{-y}\right]+y^{3 / 2} \operatorname{Ei}(-y)
\end{aligned}
$$

and $\operatorname{Ei}(-y)$ is the exponential integral. The limiting form of the function $f(y)$ for small arguments is

$$
f(y)=2 y^{1 / 2}-(0.923-\ln y) y^{3 / 2}+\ldots \quad y \rightarrow 0 \quad(y \leqslant 0.01)
$$

and the asymptotic expansion is

$$
f(y)=y^{-1 / 2} \quad y \rightarrow \infty(y \geqslant 4) .
$$

The function $f(y)$ reaches its maximum value at the point $y=0.68: f(0.68) \simeq 0.8$. A representative plot of function $f(y)$ is shown in figure 1 .

The broadening cross section, $\sigma_{v}^{\prime}$, as a function of $n^{*}$ reaches its maximum value $\sigma_{v}^{\prime \max } \simeq 2.8|L| / \bar{v}$ at the point $n^{*}=1.08(|L| / 4 \bar{v})^{1 / 4}$.

The extension of this average to the shift cross section is evident but not very necessary (see $\S 6$ ).

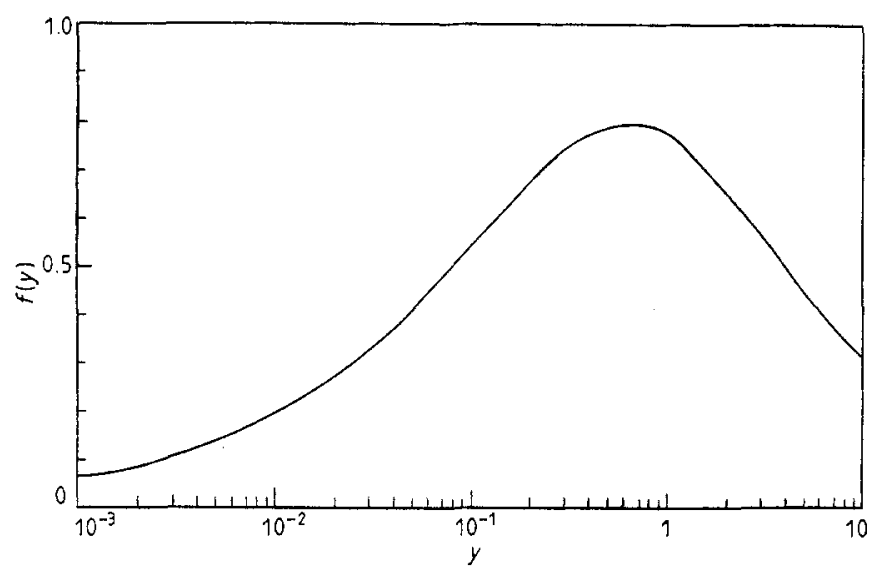

Figure 1. The function $f(y)$ according to equation (36). 


\section{High-density limit}

The impact approximation of the broadening theory used above is applicable at relatively low densities of the perturbers. The general condition for the validity of the impact approximation is a small number of perturbing particles in the interaction volume. For Rydberg-atom-neutral collisions we have two interaction distances: the Weisskopf radius for scattering by the core $\rho_{\mathrm{c}} \simeq(\pi \alpha / 4 v)^{1 / 3}$ (see equation (17)) and the radius of the Rydberg atom $r_{2}$. Thus we have as the condition of the validity of the impact approximation for the scattering by the core of the Rydberg atom

$$
\chi_{\mathrm{c}}=\frac{4}{3} \pi \rho_{\mathrm{c}}^{3} N=\left(\frac{1}{3} \pi^{2} \alpha N / v\right) \ll 1
$$

which is satisfied up to the densities $N \simeq 1 \mathrm{rd}=2.69 \times 10^{19} \mathrm{~cm}^{-3}=3.98 \times 10^{-6}$ au. For the interaction of the perturber with the Rydberg electron the condition of the validity of the impact approximation of the broadening theory formally is

$$
\chi_{\mathrm{e}}=\frac{4}{3} \pi r_{2}^{3} N \simeq \frac{32}{3} \pi n^{* 6} N \ll 1 .
$$

Note that at $N=1 \mathrm{rd}, \chi_{\mathrm{e}}=1$ when $n^{*} \simeq 4.4$.

More precisely, the expansions for the width and shift of the spectral line in powers of density are (see Royer 1980),

$$
\Delta_{\mathrm{ld}}=N d+\frac{1}{2} N^{2} a b+\ldots \quad \gamma_{\mathrm{ld}}=2 N b+\mathrm{O}\left(N^{3}\right)
$$

where $a, b$ and $d$ are constants depending on the interaction potential and velocity of the perturbers.

In the high-density limit the lineshape approaches a Gaussian and these expansions become, in powers of $N^{-1 / 2}$

$$
\begin{aligned}
& \Delta_{\mathrm{hd}}=N\langle V\rangle-\frac{1}{2}\left\langle V^{3}\right\rangle /\left\langle V^{2}\right\rangle+\mathrm{O}\left(N^{-1}\right) \\
& \gamma_{\mathrm{hd}}=N^{1 / 2} 2(2 \ln 2)^{1 / 3}\left\langle V^{2}\right\rangle^{1 / 2}+\mathrm{O}\left(N^{-1 / 2}\right)
\end{aligned}
$$

where $\langle V\rangle=\int V(\boldsymbol{R}) \mathrm{d} \boldsymbol{R}$ and classical approximation for atomic collisions is used.

In the square-well potential model (equation (31)) all quantities can be obtained in closed form. Approximately

$$
\begin{aligned}
& a=\operatorname{sgn}(L) 2^{5} \pi n^{* 10} / 3 n_{1}^{* 4} \quad b=4 \pi v n^{* 4} \quad d=0 \quad n^{*}<n_{1}^{*} \\
& a=\operatorname{sgn}(L) \frac{12 \pi n_{1}^{* 12}}{n^{* 6}}\left(1-\frac{9}{20} \frac{n_{1}^{* 8}}{n^{* 8}}\right) \quad b=\frac{9 \pi v n_{1}^{* 8}}{n^{* 4}}\left(1-\frac{1}{2} \frac{n_{1}^{* 8}}{n^{* 8}}\right) \\
& d=2 \pi L\left(1-\frac{9}{10} \frac{n_{1}^{* 8}}{n^{* 8}}\right) \quad n^{*}>n_{1}^{*} .
\end{aligned}
$$

Thus

$$
\Delta_{\mathrm{ld}} \simeq\left\{\begin{array}{l}
\operatorname{sgn}(L) \frac{2^{6} \pi^{2} v n^{* 14}}{3 n_{1}^{* 4}} N^{2} \quad n^{*}<n_{1}^{*} \\
2 \pi L\left(1-\frac{9}{10} \frac{n_{1}^{* 8}}{n^{* 8}}\right) N \\
\quad+\operatorname{sgn}(L) \frac{2 \times 3^{3} \pi^{2} v n_{1}^{*^{20}}}{n^{* 10}}\left(1-\frac{1}{2} \frac{n_{1}^{* 8}}{n^{* 8}}\right)^{2} N^{2} \quad n^{*}>n_{1}^{*}
\end{array}\right.
$$


while the expression for the width coincides with equations (32) and (1). The highdensity expansions are

$$
\begin{aligned}
& \Delta_{\mathrm{hd}}=2 \pi L N\left[1-3\left(2^{6} \pi n^{* 6} N\right)^{-1}\right] \\
& \gamma_{\mathrm{hd}}=(3 \pi \ln 2)^{1 / 2}|L| N^{1 / 2} n^{*-3} .
\end{aligned}
$$

We can formally define broadening and shift cross sections as

$$
\sigma_{v}^{\prime}=\gamma / 2 N \bar{v} \quad \sigma_{v}^{\prime \prime}=\Delta / N \bar{v}
$$

\section{Discussion and comparison with experiment}

The expressions obtained may be used for the description of the broadening and shift of $n l$ Rydberg levels by noble gases provided $l \ll n$ and the contribution from inelastic collisions to the broadening may be neglected. According to equations (26)-(30) for small $n^{*}$ broadening is defined by the Rydberg-electron-perturber interaction while for higher $n^{*}\left(n^{*} \geqslant n_{2}^{*}\right)$ the contribution of this interaction to the broadening decreases and the broadening cross section becomes defined by the core-perturber elastic scattering cross section. The Rydberg level shift for small $n^{*}$ is very small in accordance with the strong collision limit $(\eta(\rho) \gg 1)$ of the general spectral line broadening theory (see e.g. Royer 1980). Under the conditions for the Rydberg-neutral collisions with high $n^{*}$, we have the weak collision limit in the spectral line broadening theory for the Rydberg-electron-perturber interaction. We find that the shift rate due to this interaction is defined by the integral of the interaction potential and for the Fermi pseudopotential (9) equals $2 \pi L$.

If the number of perturbing atoms inside the Rydberg atom is great, i.e. if $\chi_{\mathrm{e}} \gg 1$, we must use the high-density expansions for the broadening and shift due to the Rydberg-electron-perturber interaction. In the limiting case $\chi_{\mathrm{e}} \rightarrow \infty(n \rightarrow \infty)$ the shift rate defined by this interaction approaches $2 \pi L$, the same value as in the impact approximation for high $n^{*}$, while the broadening rate decreases and is smaller than $\left\langle\sigma_{c}^{\prime} v\right\rangle$. Thus the broadening becomes defined by the Rydberg-atom-core-perturber interaction-as is also the case in the impact approximation.

Precise measurements of Rydberg level broadening until now have been performed in the relatively low-density region of the perturbing gases, i.e. at $N \ll 1 \mathrm{rd}$ while almost all the measurements of Rydberg level shifts so far have been performed in densities $N \geqslant 1$ rd (see Kaulakys and Serapinas 1984). Thus for the description of the experimental results for the broadening and shift of spectral lines associated with transitions between the ground state and highly excited states in most cases we must use the impact approximation for the broadening and high-density expansions for the shift.

In figures 2 and 3 plots of the theoretical broadening and shift cross sections are shown together with experimental results for broadening of $\mathrm{Na}(n \mathrm{~s})$ and shift of $\mathrm{Cs}(n \mathrm{p})$ levels by noble gases. Scattering lengths and polarisabilities for the rare-gas atoms employed in the present calculation are shown in table 1 . In table 1 the typical values of the critical effective principal quantum numbers $n_{1}^{*}$ and $n_{2}^{*}$ are also presented. Note that measurements of the broadening have been performed in noble gases of low density $\left(N \sim 10^{-6}-10^{-4}\right.$ rd) while measurements of the shift were carried out at $N=$ $0.948 \mathrm{rd}$. Thus for a description of the broadening we must use the impact approximation (equation (35)) but the shift of Rydberg levels with $n^{*} \leqslant 10$ produced by $\mathrm{Ar}, \mathrm{Kr}$ 


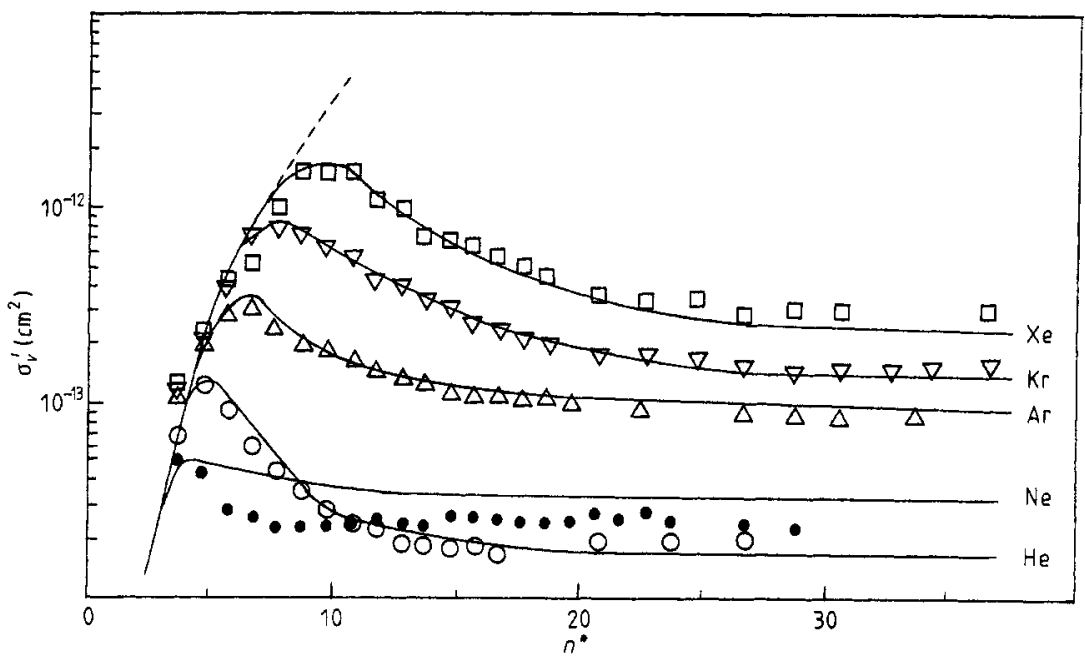

Figure 2. Broadening cross sections averaged over the Maxwellian collision velocity distribution plotted against $n^{*}$ for the broadening of $\mathrm{Na}(n \mathrm{~s})$ levels by noble gases. The symbols indicate the experimental results of Kachru et al (1980) $\left(N \sim 10^{-6}-10^{-4} \mathrm{rd}\right.$, $T=400 \mathrm{~K}$ ). The full curves show the present theoretical calculations, equation (35); the broken curve indicates $\sigma_{v}^{\prime}=4 \pi a_{0}^{2} n^{* 4}$.

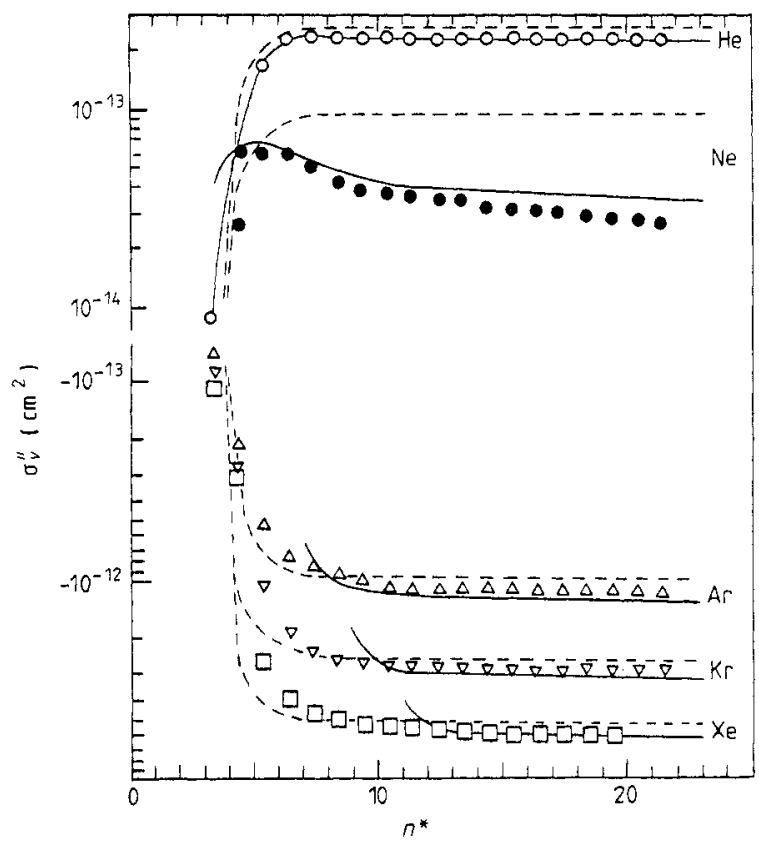

Figure 3. Shift cross sections averaged over the Maxwellian collision velocity distribution plotted against $n^{*}$ for the broadening of $\operatorname{Cs}(n \mathrm{p})$ levels by noble gases. The symbols indicate the experimental results of Tan and Ch'en $(1970)(N=0.95 \mathrm{rd}, T=550 \mathrm{~K})$. The full curves show the present calculations in the impact approximation, equation (29); the broken curves designate the high-density expansion for the square-well potential, equations (45) and (47). 
Table 1. Scattering length, $L$, and polarisability, $\alpha$, of the rare-gas atoms and typical values of the critical effective principal quantum numbers $n_{1}^{*}$ and $n_{2}^{*}$ corresponding to the collisions with $\mathrm{Na}$ at a temperature $T=400 \mathrm{~K}$.

\begin{tabular}{lcccc}
\hline Atom & $L\left(a_{0}\right)^{\mathrm{a}}$ & $\alpha\left(a_{0}^{3}\right)^{\mathrm{b}}$ & $n_{1}^{*}$ & $n_{2}^{*}$ \\
\hline $\mathrm{He}$ & 1.14 & 1.383 & 4.5 & 7.7 \\
$\mathrm{Ne}$ & 0.2 & 2.68 & 3.3 & 4.8 \\
$\mathrm{Ar}$ & -1.6 & 11.08 & 5.8 & 9.3 \\
$\mathrm{Kr}$ & -3.2 & 16.74 & 7.1 & 11.8 \\
$\mathrm{Xe}$ & -5.8 & 27.06 & 8.3 & 14.2 \\
\hline
\end{tabular}

${ }^{a}$ Golovanivsky and Kabilan (1981).

${ }^{\mathrm{b}}$ Radtsig and Smirnov (1980).

and $\mathrm{Xe}$ can only be explained by the high-density expansion (equation (45)), while for $n^{*} \gg n_{1}^{*}$ we have the weak collision limit and the expression for the shift in the high-density expansion coincides with the impact approximation. For the shift produced by $\mathrm{He}$ and $\mathrm{Ne} n_{1}^{*}$ is small $\left(n_{1}^{*}=4.4\right.$ and 3.4 respectively) and for $n^{*} \geqslant 5$ we have the weak-collision limit. That is why the shift produced by $\mathrm{He}$ and $\mathrm{Ne}$ can also be explained by the impact approximation.

The agreement of the calculation with the experiment is rather good except for broadening by neon for which the scattering length approximation is not very suitable. For helium this approximation is valid if the electron's energy is smaller than about $1 \mathrm{eV}$ (see e.g. Golovanivsky and Kabilan 1981). The scattering length approximation for the description of the scattering of the electrons by heavy rare-gas atoms is appropriate for very low energy, or slow, electrons. At large distances from the core the Rydberg electron is slow and that is why the Fermi pseudopotential approximation (equation (9)) for the interaction of the Rydberg electron with rare-gas atoms is suitable at large impact parameters. On the other hand, the broadening and shift cross sections (see equations (2) and (3)) depend mainly on the phaseshift $\eta(\rho)$ at values of $\rho$ for which $\eta(\rho) \leqslant 1$. However, according to equation (19) such a condition at small $n^{*}$ is valid for large $\rho$. Therefore our calculations are valid for the description of the broadening and shift of the Rydberg levels with small, as well as with large, $n^{*}$ by all rare-gas atoms except neon. The theory must be improved when inelastic collisions are important (see Gounand et al 1982, Kaulakys and Serapinas 1984).

Finally a few remarks should be made on recent papers that have treated Rydbergneutral collisions. Thus in papers by Hahn (1981, 1982), Flannery (1982) and Matsuzawa (1984) the impulse approximation theory has been developed and the limits of applicability of the impulse approximation in thermal Rydberg-neutral collisions has been discussed. In a paper by Cheng and van Regemorter (1981) semianalytical methods are presented for computing quantum form factors in inelastic Rydbergneutral collisions. Model potential calculations for the Rydberg states of the $\mathrm{NaNe}$ molecule have been performed in a paper by Masnou-Seeuws et al (1982) which predicted that rare-gas collisions could induce a population transfer from a Rydberg $(n+1)$ p level to the $n l(l \geqslant 2)$ manifold. Such transitions would not be predicted by the Fermi model. However, as is shown in the present paper, such complicated numerical calculations are not necessary for the description of the broadening and shift of Rydberg levels by all rare gases excepting neon when the contribution from inelastic collisions is negligible. That is why the broadening and shift cross sections are not very sensitive to the dependence of the Rydberg-electron-perturber interaction 
potential on the internuclear distance and angular coordinates but are defined by the integral of the interaction potential (see $\$ 4$ ).

Summarising, expressions for the Rydberg level impact broadening and shift cross sections suitable for elastic collisions of ground-state rare-gas atoms with Rydberg atoms have been obtained. The potential of the interaction of a Rydberg atom with a rare-gas atom is approximated by a superposition of polarisation potentials and the Fermi pseudopotential. Similar expressions of the broadening and shift cross sections have been obtained for the scattering by the square-well potential and it was shown that such expressions are not very sensitive to the shape of the potential but only to its normalisation. This allowed us to use the square-well potential for the construction of the high-density expansions for the width and shift of the Rydberg levels. It was shown that low-density and high-density expansions for high $n\left(n^{*} \gg n_{2}^{*}\right)$ lead to the same broadening and shift rates while for small and medium $n$ non-linear dependences of the broadening and shift on the perturbing gas density were predicted.

\section{References}

Alekseev V V and Sobel'man I I 1965 Zh. Eksp. Teor. Fiz. 49 1274-83 (1966 Sov. Phys.-JETP 22 882-8)

Baylis W E 1969 J. Chem. Phys. 51 2665-79

Ch'en S and Takeo M 1957 Rev. Mod. Phys. 29 20-73

Cheng L Y and van Regemorter H 1981 J. Phys. B: At. Mol. Phys. 14 4025-40

Fermi E 1934 Nuovo Cim. 11 157-66

Firsov O B 1951 Zh. Eksp. Teor. Fiz. 21 627-33, 634-41

Flannery M R 1982 J. Phys. B: At. Mol. Phys. 15 3249-56

Golovanivsky K S and Kabilan A P 1981 Zh. Eksp. Teor. Fiz. 80 2210-20

Gounand F, Szudy J, Hugon M, Sayer B and Fournier P R 1982 Phys. Rev. A $26831-41$

Hahn Y 1981 J. Phys. B: At. Mol. Phys. $14985-96$

1982 J. Phys. B: At. Mol. Phys. 15 613-23

Hickman A P 1979 Phys. Rev. A $19994-1003$

Kachru R, Mossberg T W and Hartmann S R 1980 Phys. Rev. A 21 1124-33

Kaulakys B P 1980 Opt. Spektrosk. 48 1047-53 (1981 Opt. Spectrosc. 48 574-7)

- 1982 J. Phys. B: At. Mol. Phys. 15 L719-22

Kaulakys B P, Presnyakov L P and Serapinas P D 1979 Zh. Eksp. Teor. Fiz. Pis. 30 60-3 (1980 JETP Lett. 30 53-5)

Kaulakys B P and Serapinas P D 1984 Liet. Fiz. Rinkinys 24 N3 3-37 (English transl. Soviet Physics Collection: Litovskii Fizicheskii Sboznik (New York: Allerton))

Landau L D and Lifshitz E M 1960 Quantum Mechanics (Oxford: Pergamon)

Masnou-Seeuws F, Boulmer J, Maurin T, Roche A L and Valiron P 1982 J. Phys. B: At. Mol. Phys. 15 2989-3003

Matsuzawa M 1975 J. Phys. B: At. Mol. Phys. 8 L382-6

1977 J. Phys. B: At. Mol. Phys. $101543-56$

1984 J. Phys. B: At. Mol. Phys. 17 795-810

Mazing M A and Serapinas P D 1971 Zh. Eksp. Teor. Fiz. $60541-51$ (1971 Sov. Phys.-JETP 33 294)

Omont A 1977 J. Physique 38 1343-59

Radtsig A A and Smirnov B M 1980 Handbook on Atomic and Molecular Physics (in Russian) (Moscow: Atomizdat)

Reinsberg C 1937 Z. Phys. 105 460-7

Royer A 1980 Phys. Rev. A 22 1625-54

Sobel'man I I, Wainshtein L A and Yukov E A 1981 Excitation of Atoms and Broadening of Spectral Lines (Berlin: Springer)

Stoicheff B P, Thomson D C and Weinberger E 1981 Spectral Line Shapes, Proc. Sth Int. Conf., Berlin 1980 ed B Wendt (Berlin: de Gruyter) pp 1071-82

Stoicheff B P and Weinberger E 1980 Phys. Rev. Lett. 44 733-6

Tan D K L and Ch'en S Y 1970 Phys. Rev. A $21124-9$

Ueda K, Hamaguchi Y and Fukuda K 1982a J. Phys. Soc. Japan 51 13-4

— 1982b J. Phys. Soc. Japan 51 1948-52 